\section{Curative Evaluation of Biological Control Agents and Synthetic Fungicides for Clarireedia jacksonii}

\author{
Jeffery W. Marvin \\ PBI-Gordon Corporation, Shawnee, KS 66226
}

Robert Andrew Kerr
Chicago District Golf Association, Lemont, IL 60439

Lambert B. McCarty

Department of Plant, and Environmental Sciences, Clemson University, 130 McGinty Court, Clemson, SC 29634

William Bridges
Department of Mathematical Sciences, Clemson University, Martin O-117,
Clemson, SC 29634

S. Bruce Martin

Department of Plant, and Environmental Sciences, Clemson University, 2200

Pocket Road, Darlington, SC 29532

Christina E. Wells

Department of Biological Sciences, Clemson University, 132 Long Hall, Clemson, SC 29634

Additional index words. Agrostis stolonifera L. var. palustris (Huds) cv. Crenshaw, Clarireedia jacksonii sp. nov., dollar spot, Sclerotinia homoeocarpa F.T. Bennett, turfgrass disease

\begin{abstract}
Clarireedia jacksonii sp. nov. formerly Sclerotinia homoeocarpa F.T. Bennett, one of the causal agents of dollar spot, is the most widespread pathogen in turfgrass systems. Dollar spot (DS) affects both cool- and warm-season grasses, during a wide range of environmental conditions. Field studies were conducted at Clemson University, Clemson, SC, on a creeping bentgrass [Agrostis stolonifera L. var. palustris (Huds) cv. Crenshaw] putting green for 2 consecutive years from August to October in year 1 and July to September in year 2. The objective of the studies was to evaluate biological control agents (BCAs) and synthetic fungicides at reduced rates for their efficacy controlling dollar spot. Four replications of $1.5 \times 1.5-\mathrm{m}$ plots were used in the experimental design. Treatments included the following: Bacillus subtilis (BS); plant extract oils (EO) including clove oil + wintergreen oil + thyme oil; extract of Reynoutria sachalinensis (RS); Bacillus licheniformis (BL); chlorothalonil (CL); and azoxystrobin + propiconazole (AzP). Synthetic fungicides were used at reduced rates in combination with biological control agents, to evaluate curative control efficacy of various combinations. All reduced synthetic programs, except CL + EO, provided acceptable disease severity $(\leq 15 \%)$ at the end of year 1 and acceptable $(\geq 7)$ turfgrass visual quality. Azoxystrobin + propiconazole, CL, AzP + BL, AzP + EO, AzP + BS all provided $\leq 15 \%$ disease severity and $\geq 7$ visual turfgrass quality 14 days after the last application in year 2 .
\end{abstract}

C. jacksonii sp. nov. formerly S. homoeocarpa F.T. Bennett, the causal agent of DS, is the most widespread pathogen in turfgrass systems (Bishop et al., 2008; Salgado-Salazar et al., 2018). DS affects both cool- and warmseason grasses, during a wide range of envi-

Received for publication 16 Apr. 2020. Accepted for publication 15 July 2020 .

Published online 21 August 2020.

R.A.K. is the corresponding author. E-mail: rakerr@ g.clemson.edu.

This is an open access article distributed under the CC BY-NC-ND license (https://creativecommons. org/licenses/by-nc-nd/4.0/). ner has reinvigorated the search for suitable biological fungicides. Aiding the drive are costs associated with synthetic fungicides and perceived risks to humans and the environment.

As an alternative to synthetic products, bacterial and fungal species are being evaluated for their ability to suppress DS. BCAs offer both advantages and disadvantages to turf managers. Potential biological species for use are native to most soils, and thus pose low toxicity risk to humans and wildlife. Synthetic products are subject to degradation, where active parent products are degraded into secondary metabolites that may pose certain risks (Harman, 2006). However, currently available BCAs typically show reduced acute efficacy and residual control. Application timing becomes much more critical, especially during high disease pressure conditions. Packaging and shelf life issues also pose challenges. Most of these products contain living organisms, requiring shortterm storage under specific conditions (Kim et al., 1997). In this study, the goal was to investigate if quarter low label rates of the synthetic fungicides $\mathrm{CL}$ and $\mathrm{AzP}$ in combination with BCAs could provide similar control as full label rates of the synthetic fungicides.

\section{Materials and Methods}

Studies were conducted at Clemson University, Clemson, SC (lat. $34.67^{\circ} \mathrm{N}$, long. $82.84^{\circ} \mathrm{W}$ ) on a creeping bentgrass [Agrostis stolonifera L. var. palustris (Huds) cv. Crenshaw] putting green from Aug. to Oct. 2008 (year 1) and July to Sept. 2009 (year 2). The research site was an 11-year-old 85:15 U.S. Golf Association sand:peat-based green construction. Crenshaw creeping bentgrass was selected due to its high susceptibility to DS (McCarty, 2018).

Mowing height was $\approx 3 \mathrm{~mm}$ and occurred 5 to 7 times weekly. Irrigation was applied as needed to prevent moisture stress. Nitrogen was applied annually at $1.81 \mathrm{~kg} \cdot \mathrm{ha}^{-1} \mathrm{~N}$. Nutrients were supplied via organic-based products using granular and liquid products. Additional potassium $\left(\mathrm{K}_{2} \mathrm{O}\right)$ and phosphorous $\left(\mathrm{P}_{2} \mathrm{O}_{5}\right)$ was applied through separate products to provide a $1-1-2$ ratio of $\mathrm{N}-\mathrm{P}-$ K. Fertility treatments included EndoRoots 3-3-5 (LebanonTurf, Lebanon, PA) at 0.23 $\mathrm{kg} \cdot \mathrm{ha}^{-1} \mathrm{~N}$ monthly, and Novozymes Turf Vigor 9-3-6 (LebanonTurf) was applied at $0.11 \mathrm{~kg} \cdot \mathrm{ha}^{-1} \mathrm{~N}$ biweekly.

Four replications of $1.5 \times 1.5-\mathrm{m}$ plots were used in the experimental design. Spray applications were made using a pressurized $\mathrm{CO}_{2}$ backpack boom sprayer, through 8003 flat-fan nozzles (Tee jet; Spraying Systems Co., Roswell, GA) with a water carrier volume of $374 \mathrm{~L} \cdot \mathrm{ha}^{-1}$. Products were applied in cooler morning or evening hours to minimize turfgrass phytotoxicity. Treatments included BCAs and conventional control products (Table 1).

Reduced synthetics. Programs were based on applications of CL (Daconil Ultrex 
Table 1. Fungicide common name, trade name, and manufacturer of products used during a 2-year study on Crenshaw creeping bentgrass for curative control and suppression of dollar spot (Clarireedia jacksonii).

\begin{tabular}{|c|c|c|}
\hline Common name & Trade name & Manufacturer \\
\hline$\overline{\text { Azoxystrobin + propiconazole }}$ & Headway $1.39 \mathrm{SC}$ & Syngenta, Greensboro, NC \\
\hline Chlorothalonil & Daconil Ultrex 82.5WP & Syngenta, Greensboro, NC \\
\hline Bacillus licheniformis SB3086 & EcoGuard $0.36 \mathrm{SC}$ & Novozymes, Salem, VA \\
\hline Reynoutria sachalinensis extract & Regalia $0.48 \mathrm{SC}$ & Marrone Bio Innovations, Davis, CA \\
\hline Bacillus subtilis QST 713 & Rhapsody $0.13 \mathrm{SC}$ & AgraQuest, Davis, CA \\
\hline Clove oil, thyme oil, and rosemary oil & Paradigm $3.61 \mathrm{~L}$ & Parkway Research, Pleasant Plains, II \\
\hline
\end{tabular}

Table 2. Treatments evaluated during a 2-year study for the efficacy of combinations of BCAs and synthetic products for dollar spot control on Crenshaw creeping bentgrass.

\begin{tabular}{|c|c|c|c|c|}
\hline Treatment & Treatment abbreviation & Rate $\left(\mathrm{L} \cdot \mathrm{ha}^{-1}\right)$ & Application & Total Applications \\
\hline Azoxystrobin + propiconazole & $\mathrm{AzP}$ & 4.77 & Biweekly & 6 \\
\hline Azoxystrobin + propiconazole + Bacillus licheniformis & $\mathrm{AzP}+\mathrm{BL}$ & 2.39 and 63.6 & Biweekly & 6 \\
\hline Azoxystrobin + propiconazole + Reynoutria sachalinensis & $\mathrm{AzP}+\mathrm{RS}$ & 2.39 and $5 \% \mathrm{v} / \mathrm{v}$ & Biweekly & 6 \\
\hline Azoxystrobin + propiconazole + Bacillus subtilis & $\mathrm{AzP}+\mathrm{BS}$ & 2.39 and 31.8 & Biweekly & 6 \\
\hline Azoxystrobin + propiconazole + clove, thyme, and rosemary oil & $\mathrm{AzP}+\mathrm{EO}$ & 2.39 and 9.54 & Biweekly & 6 \\
\hline Chlorothalonil & $\mathrm{CL}$ & 9.54 & Biweekly & 6 \\
\hline Chlorothalonil + Bacillus licheniformis & $\mathrm{CL}+\mathrm{BL}$ & 2.39 and 63.6 & Biweekly & 6 \\
\hline Chlorothalonil + Reynoutria sachalinensis & $\mathrm{CL}+\mathrm{RS}$ & 2.39 and $5 \% \mathrm{v} / \mathrm{v}$ & Biweekly & 6 \\
\hline Chlorothalonil + Bacillus subtilis & $\mathrm{CL}+\mathrm{BS}$ & 2.39 and 31.8 & Biweekly & 6 \\
\hline Chlorothalonil + clove, thyme, and rosemary oil & $\mathrm{CL}+\mathrm{EO}$ & 2.39 and 9.54 & Biweekly & 6 \\
\hline
\end{tabular}

Table 3. Visual quality on Crenshaw creeping bentgrass in year 1 from a curative dollar spot study. Study initiated 30 July 2008.

\begin{tabular}{|c|c|c|c|c|c|c|}
\hline \multirow[b]{2}{*}{ Treatment } & \multicolumn{6}{|c|}{ Visual quality ${ }^{z}$} \\
\hline & 6 Aug. & 20 Aug. & 3 Sept. & 17 Sept. & 1 Oct. & 15 Oct. \\
\hline Azoxystrobin + propiconazole & $3.0 \mathrm{a}^{\mathrm{y}}$ & $3.5 \mathrm{ab}$ & $6.2 \mathrm{a}$ & $7.2 \mathrm{a}$ & $7.7 \mathrm{ab}$ & $8.2 \mathrm{a}$ \\
\hline Azoxystrobin + propiconazole + Bacillus licheniformis & $3.0 \mathrm{a}$ & $3.3 \mathrm{ab}$ & $4.5 \mathrm{bc}$ & $7 \mathrm{ab}$ & $7.8 \mathrm{a}$ & $8 \mathrm{ab}$ \\
\hline Azoxystrobin + propiconazole + Reynoutria sachalinensis & $3.0 \mathrm{a}$ & $3.3 \mathrm{ab}$ & $4.3 \mathrm{bcd}$ & $6.2 \mathrm{~cd}$ & $7 \mathrm{bc}$ & $7.3 \mathrm{de}$ \\
\hline Azoxystrobin + propiconazole + Bacillus subtilis & $3.0 \mathrm{a}$ & $3 \mathrm{~b}$ & $4.5 \mathrm{bc}$ & $6.8 \mathrm{abc}$ & $7.2 \mathrm{abc}$ & $7.5 \mathrm{~cd}$ \\
\hline Azoxystrobin + propiconazole + clove, thyme, and rosemary oil & $3.0 \mathrm{a}$ & $3.3 \mathrm{ab}$ & $4.8 \mathrm{~b}$ & $5.8 \mathrm{~d}$ & $7 \mathrm{bc}$ & $7.5 \mathrm{~cd}$ \\
\hline Chlorothalonil & $3.0 \mathrm{a}$ & $3.3 \mathrm{ab}$ & $6.5 \mathrm{a}$ & $6.8 \mathrm{abc}$ & $7.3 \mathrm{ab}$ & $7.8 \mathrm{abc}$ \\
\hline Chlorothalonil + Bacillus licheniformis & $3.0 \mathrm{a}$ & $3.7 \mathrm{a}$ & $4.3 \mathrm{bcd}$ & $6.3 \mathrm{bcd}$ & $7.2 \mathrm{abc}$ & $7.5 \mathrm{~cd}$ \\
\hline Chlorothalonil + Reynoutria sachalinensis & $3.0 \mathrm{a}$ & $3.2 \mathrm{ab}$ & 4 bcd & $5.7 \mathrm{~d}$ & $6.2 \mathrm{~d}$ & $7 \mathrm{e}$ \\
\hline Chlorothalonil + Bacillus subtilis & $3.0 \mathrm{a}$ & $3 \mathrm{~b}$ & $3.7 \mathrm{~cd}$ & $5.7 \mathrm{~d}$ & $6.5 \mathrm{~cd}$ & $7.7 \mathrm{bcd}$ \\
\hline Chlorothalonil + clove, thyme, and rosemary oil & $3.0 \mathrm{a}$ & $3.2 \mathrm{ab}$ & $3.5 \mathrm{~d}$ & $4.2 \mathrm{e}$ & $5.2 \mathrm{e}$ & $5.5 \mathrm{f}$ \\
\hline
\end{tabular}

${ }^{\mathrm{z}}$ Visual quality was rated on a scale of 1 to $9: 1=$ dead turf, $9=$ perfect turf, and $7=$ minimum acceptable.

${ }^{\mathrm{y}}$ Means with the same letter are not significantly different based on Fisher's least significant difference test $(\alpha=0.05)$.

82.5WP) or AzP (Headway 1.39SC) every $14 \mathrm{~d}$ at quarter label rates and tank mixed with the designated BCA. Chlorothalonil (2 $\mathrm{kg}$ a.i./ha) and $\mathrm{AzP}(0.4 \mathrm{~kg}$ a.i./ha) were considered quarter label rate. Biological control agents were BS formulated as Rhapsody SC; EO clove oil + wintergreen oil + thyme oil formulated as Paradigm L; extract of RS formulated as Regalia L; and BL formulated as EcoGuard SC (Table 2).

Synthetics. Synthetic products, CL $(8.2 \mathrm{~kg}$ a.i./ha) and AzP (1.6 kg a.i./ha) were applied every $14 \mathrm{~d}$ at full label rate. Synthetic programs were the industry standards and BCA treatments were compared (Table 2).

Turfgrass plots were rated weekly for disease severity and visual turfgrass quality. Disease severity ratings used a line intersect grid rating method. Each plot was divided into 289 smaller subplots considered a grid, measuring $100 \times 100 \mathrm{~mm}$. Disease in any portion of a grid was considered a "hit." A percentage of disease severity was calculated by taking the number of hits and dividing by the total number of grids multiplied by 100 . Severity ratings more aptly show the level of fungicidal activity provided by the various treatments. An acceptable disease severity level was set as $\leq 15 \%$.

Turfgrass visual quality was rated on a scale of 1 to 9 , where $1=$ brown dead turf, $7=$ minimum accepted, and $9=$ dark green dense turf (Morris and Shearman, 1999). Weekly ratings were averaged each month for statistical analysis.

Areas under disease progress curve (AUDPC) were calculated for each fungicide and rate using the following equation:

$$
\mathrm{AUDPC}=\sum\left[\left(\mathrm{Y}_{1}+\mathrm{Y}_{2}\right) / 2\right]\left(\mathrm{T}_{2}-\mathrm{T}_{1}\right),
$$

where $Y$ equals rating date and $T$ equals days between rating date (Latin, 2008). The AUDPC calculates the disease epidemic over the course of the rating period.

Statistical analysis. The SAS statistical software package JMP Pro 9.1 (SAS Institute Inc., Cary, NC) was used for analysis of variance (ANOVA) and means separation on all data sets. The ANOVA was used to evaluate the main effects of experimental run per year, fungicide program, and days after initiation, as well as the interactions. When the main effects or interactions were significant, Fisher's least significant difference test $(\alpha=0.05)$ was used to separate means.

\section{Results}

Analysis of variance indicated an interaction of experimental run and year; therefore, data were not combined between years and is presented separately. Experimental run per year was part of the statistical model.

Turfgrass visual quality. In year 1, no differences occurred on 6 Aug. or 20 Aug. $(\approx 3)$ (Table 3). On 3 Sept., AzP and CL had greater turfgrass visual quality $(>6)$, compared with the reduced synthetic programs $(\approx 4.5)$ (Table 3). On 17 Sept., AzP had the highest turfgrass visual quality $(\approx 7.2)$, followed by $\mathrm{AzP}+\mathrm{BL}(\approx 7), \mathrm{AzP}+\mathrm{BS}$ and $\mathrm{CL}$ $(\approx 6.8), \mathrm{CL}+\mathrm{BL}(\approx 6.3), \mathrm{AzP}+\mathrm{RS}(\approx 6.2)$, $\mathrm{AzP}+\mathrm{EO}(\approx 5.8), \mathrm{CL}+\mathrm{RS}$ and $\mathrm{CL}+\mathrm{BS}$ $(\approx 5.7)$, and CL + EO (4.2) (Table 3). On Oct. $1, \mathrm{AzP}, \mathrm{AzP}+\mathrm{BL}, \mathrm{AzP}+\mathrm{RS}, \mathrm{AzP}+\mathrm{BS}, \mathrm{AzP}+$ $\mathrm{EO}, \mathrm{CL}$, and $\mathrm{CL}+\mathrm{BL}$ experienced highest turfgrass visual quality $(>\approx 7)$; compared with $\mathrm{CL}+\mathrm{RS}, \mathrm{CL}+\mathrm{BS}(<\approx 6.5)$, and $\mathrm{CL}+$ $\mathrm{EO}(\approx 5.2)$ (Table 3). On Oct. 15, AzP, AzP + $\mathrm{BL}, \mathrm{CL}$ experienced highest turfgrass visual quality $(>\approx 7.8)$, followed by AzP + RS, AzP + $\mathrm{BS}, \mathrm{AzP}+\mathrm{EO}, \mathrm{CL}+\mathrm{BS}(\approx 7.5) ; \mathrm{CL}+\mathrm{RS}$ $(\approx 7)$, and $\mathrm{CL}+\mathrm{EO}(\approx 5.5)($ Table 3$)$.

In year 2 , no differences occurred on 1 July or 14 July $(\approx 3)$ (Table 4$)$. On 4 Aug., AzP and CL had greater turfgrass visual quality $(\approx 7)$, compared with the reduced synthetic programs $(>\approx 4.7)$ (Table 4$)$. On 18 Aug., AzP, AzP + BL and CL experienced highest turfgrass visual quality $(\approx 7.3)$, followed by AzP + BS and AzP + EO $(\approx 6.7)$, 


\begin{tabular}{|c|c|c|c|c|c|c|}
\hline \multirow[b]{2}{*}{ Treatment } & \multicolumn{6}{|c|}{ Visual quality ${ }^{z}$} \\
\hline & 1 July & 14 July & 4 Aug. & 18 Aug. & 31 Aug. & 11 Sept \\
\hline Azoxystrobin + propiconazole & $3.0 \mathrm{a}^{\mathrm{y}}$ & $3.0 \mathrm{~b}$ & $7.0 \mathrm{a}$ & $7.3 \mathrm{a}$ & $7.7 \mathrm{a}$ & $7.8 \mathrm{a}$ \\
\hline Azoxystrobin + propiconazole + Bacillus licheniformis & $3.0 \mathrm{a}$ & $3.0 \mathrm{~b}$ & $4.7 \mathrm{~b}$ & $7.2 \mathrm{ab}$ & $7.6 \mathrm{a}$ & $7.3 \mathrm{a}$ \\
\hline Azoxystrobin + propiconazole + Reynoutria sachalinensis & $3.0 \mathrm{a}$ & $3.0 \mathrm{~b}$ & $4.2 \mathrm{bc}$ & $6.5 \mathrm{~cd}$ & $6.8 \mathrm{~b}$ & $5.3 \mathrm{~b}$ \\
\hline Azoxystrobin + propiconazole + Bacillus subtilis & $3.0 \mathrm{a}$ & $3.0 \mathrm{~b}$ & $4.2 \mathrm{bc}$ & $6.7 \mathrm{bc}$ & $7.0 \mathrm{~b}$ & $5.7 \mathrm{~b}$ \\
\hline Azoxystrobin + propiconazole + clove, thyme, and rosemary oil & $3.0 \mathrm{a}$ & $3.0 \mathrm{~b}$ & $4.7 \mathrm{~b}$ & $6.7 \mathrm{bc}$ & $6.8 \mathrm{~b}$ & $5.8 \mathrm{~b}$ \\
\hline Chlorothalonil & $3.0 \mathrm{a}$ & $3.0 \mathrm{~b}$ & $6.7 \mathrm{a}$ & $7.3 \mathrm{a}$ & $7.5 \mathrm{a}$ & $6.0 \mathrm{~b}$ \\
\hline Chlorothalonil + Bacillus licheniformis & $3.0 \mathrm{a}$ & $3.2 \mathrm{ab}$ & $4.2 \mathrm{bc}$ & $6.0 \mathrm{de}$ & $6.7 \mathrm{~b}$ & $5.3 \mathrm{~b}$ \\
\hline Chlorothalonil + Reynoutria sachalinensis & $3.0 \mathrm{a}$ & $3.0 \mathrm{~b}$ & $3.8 \mathrm{~cd}$ & $6.5 \mathrm{~cd}$ & $5.5 \mathrm{~d}$ & $4.5 \mathrm{~b}$ \\
\hline Chlorothalonil + Bacillus subtilis & $3.0 \mathrm{a}$ & $3.0 \mathrm{~b}$ & $3.5 \mathrm{~cd}$ & 5.7 ef & $6.0 \mathrm{c}$ & $3.5 \mathrm{~d}$ \\
\hline Chlorothalonil + clove, thyme, and rosemary oil & $3.0 \mathrm{a}$ & $3.0 \mathrm{~b}$ & $3.3 \mathrm{~d}$ & $5.2 \mathrm{f}$ & $6.8 \mathrm{~b}$ & $3.5 \mathrm{~d}$ \\
\hline
\end{tabular}

${ }^{\mathrm{z}}$ Visual quality was rated on a scale of 1 to $9: 1=$ dead turf, $9=$ perfect turf, and $7=$ minimum acceptable.

${ }^{\mathrm{y}}$ Means with the same letter are not significantly different based on Fisher's least significant difference test $(\alpha=0.05)$.

Table 5. Disease severity on Crenshaw creeping bentgrass in year 1 from a curative dollar spot study. Study initiated 30 July 2008.

\begin{tabular}{|c|c|c|c|c|c|c|}
\hline \multirow[b]{2}{*}{ Treatment } & \multicolumn{6}{|c|}{ Disease severity $^{z}$} \\
\hline & 6 Aug. & 20 Aug. & 3 Sept. & 17 Sept. & 1 Oct. & 15 Oct \\
\hline Azoxystrobin + propiconazole & $99.7 \mathrm{a}^{\mathrm{y}}$ & 92.4 bc & $36.0 \mathrm{~b}$ & $6.6 \mathrm{e}$ & $2.1 \mathrm{de}$ & $0.3 \mathrm{c}$ \\
\hline Azoxystrobin + propiconazole + Bacillus licheniformis & $98.3 \mathrm{a}$ & $96.1 \mathrm{abc}$ & $71.5 \mathrm{a}$ & $10.6 \mathrm{de}$ & $1.4 \mathrm{e}$ & $0.7 \mathrm{c}$ \\
\hline Azoxystrobin + propiconazole + Reynoutria sachalinensis & $98.2 \mathrm{a}$ & $95.9 \mathrm{abc}$ & $84.5 \mathrm{a}$ & $24.6 \mathrm{cbd}$ & $13.3 \mathrm{c}$ & $3.1 \mathrm{c}$ \\
\hline Azoxystrobin + propiconazole + Bacillus subtilis & $99.6 \mathrm{a}$ & $94.6 \mathrm{abc}$ & $80.0 \mathrm{a}$ & 19.8 cde & 8.6 cde & $2.7 \mathrm{c}$ \\
\hline Azoxystrobin + propiconazole + clove, thyme, and rosemary oil & $98.8 \mathrm{a}$ & $97.2 \mathrm{a}$ & $73.8 \mathrm{a}$ & $34.1 \mathrm{bc}$ & $15.2 \mathrm{bc}$ & $3.1 \mathrm{c}$ \\
\hline Chlorothalonil & $97.7 \mathrm{a}$ & $92.3 \mathrm{bc}$ & $35.6 \mathrm{~b}$ & $8.5 \mathrm{e}$ & $1.8 \mathrm{de}$ & $0.3 \mathrm{c}$ \\
\hline Chlorothalonil + Bacillus licheniformis & $97.4 \mathrm{a}$ & $91.8 \mathrm{c}$ & $68.8 \mathrm{a}$ & 19.6 cde & $11.8 \mathrm{cde}$ & $3.6 \mathrm{c}$ \\
\hline Chlorothalonil + Reynoutria sachalinensis & $97.0 \mathrm{a}$ & $93.3 \mathrm{abc}$ & $83.3 \mathrm{a}$ & $35.5 \mathrm{~b}$ & $25.7 \mathrm{~b}$ & $12.1 \mathrm{~b}$ \\
\hline Chlorothalonil + Bacillus subtilis & $98.0 \mathrm{a}$ & $93.8 \mathrm{abc}$ & $88.2 \mathrm{a}$ & $34.9 \mathrm{bc}$ & $12.4 \mathrm{~cd}$ & $4.4 \mathrm{c}$ \\
\hline Chlorothalonil + clove, thyme, and rosemary oil & $97.9 \mathrm{a}$ & $96.7 \mathrm{ab}$ & $90.6 \mathrm{a}$ & $74.6 \mathrm{a}$ & $65.5 \mathrm{a}$ & $42.5 \mathrm{a}$ \\
\hline
\end{tabular}

${ }^{\mathrm{z}}$ Disease severity was determined using a grid intersect on $3 \mathrm{~m}^{2}$ plots, with $15 \%=$ maximum acceptable disease severity rating.

${ }^{\mathrm{y}}$ Means with the same letter are not significantly different based on Fisher's least significant difference test $(\alpha=0.05)$.

Table 6. Disease severity on Crenshaw creeping bentgrass in year 2 from a curative dollar spot study. Study initiated 25 June 2009.

\begin{tabular}{|c|c|c|c|c|c|c|}
\hline \multirow[b]{2}{*}{ Treatments } & \multicolumn{6}{|c|}{ Disease severity $^{z}$} \\
\hline & 1 July & 14 July & 4 Aug. & 18 Aug. & 31 Aug. & 11 Sept. \\
\hline Azoxystrobin + propiconazole & $100 \mathrm{a}^{\mathrm{y}}$ & $94.7 \mathrm{c}$ & $5.8 \mathrm{e}$ & $1.5 \mathrm{~d}$ & $1.0 \mathrm{e}$ & $1.0 \mathrm{f}$ \\
\hline Azoxystrobin + propiconazole + Bacillus licheniformis & $100 \mathrm{a}$ & $96.9 \mathrm{abc}$ & $84.6 \mathrm{c}$ & $4.6 \mathrm{~d}$ & $3.1 \mathrm{de}$ & 9.5 ef \\
\hline Azoxystrobin + propiconazole + Reynoutria sachalinensis & $100 \mathrm{a}$ & $96.7 \mathrm{abc}$ & $93.9 \mathrm{ab}$ & $20.2 \mathrm{c}$ & $17.5 \mathrm{bc}$ & $44.6 \mathrm{c}$ \\
\hline Azoxystrobin + propiconazole + Bacillus subtilis & $100 \mathrm{a}$ & $95.4 \mathrm{bc}$ & $94.5 \mathrm{ab}$ & $13.9 \mathrm{c}$ & $10.4 \mathrm{~cd}$ & $35.1 \mathrm{~d}$ \\
\hline Azoxystrobin + propiconazole + clove, thyme, and rosemary oil & $100 \mathrm{a}$ & $97.8 \mathrm{abc}$ & $79.8 \mathrm{c}$ & $18.4 \mathrm{c}$ & $14.7 \mathrm{c}$ & $53.5 \mathrm{bc}$ \\
\hline Chlorothalonil & $100 \mathrm{a}$ & $95.6 \mathrm{abc}$ & $17.5 \mathrm{~d}$ & $5.5 \mathrm{~d}$ & $1.4 \mathrm{de}$ & $18.2 \mathrm{e}$ \\
\hline Chlorothalonil + Bacillus licheniformis & $100 \mathrm{a}$ & $96.9 \mathrm{abc}$ & $88.7 \mathrm{bc}$ & $20.5 \mathrm{c}$ & $18.8 \mathrm{bc}$ & $58.7 \mathrm{~b}$ \\
\hline Chlorothalonil + Reynoutria sachalinensis & $100 \mathrm{a}$ & $99.7 \mathrm{a}$ & $94.0 \mathrm{ab}$ & $45.3 \mathrm{a}$ & $40.3 \mathrm{a}$ & $85.6 \mathrm{a}$ \\
\hline Chlorothalonil + Bacillus subtilis & $100 \mathrm{a}$ & $98.9 \mathrm{abc}$ & $96.4 \mathrm{ab}$ & $32.3 \mathrm{~b}$ & $24.2 \mathrm{~b}$ & $90.9 \mathrm{a}$ \\
\hline Chlorothalonil + clove, thyme, and rosemary oil & $100 \mathrm{a}$ & $97.8 \mathrm{abc}$ & $98.4 \mathrm{a}$ & $50.3 \mathrm{a}$ & $43.2 \mathrm{a}$ & $91.5 \mathrm{a}$ \\
\hline
\end{tabular}

${ }^{\mathrm{z}}$ Disease severity was determined using a grid intersect on $3 \mathrm{~m}^{2}$ plots, with $15 \%=$ maximum acceptable disease severity rating.

${ }^{\mathrm{y}}$ Means with the same letter are not significantly different based on Fisher's least significant difference test $(\alpha=0.05)$.

$\mathrm{AzP}+\mathrm{RS}$ and $\mathrm{CL}+\mathrm{RS}(\approx 6.5), \mathrm{CL}+\mathrm{BL}$ $(\approx 6), \mathrm{CL}+\mathrm{BS}(\approx 5.7)$, and $\mathrm{CL}+\mathrm{EO}(\approx 5.2)$ (Table 4). On 31 Aug., AzP, AzP + BL and CL experienced highest turfgrass visual quality $(>\approx 7.5)$; followed by $\mathrm{AzP}+\mathrm{BS}(\approx 7), \mathrm{AzP}+$ $\mathrm{RS}, \mathrm{AzP}+\mathrm{EO}$, and $\mathrm{CL}+\mathrm{EO}(\approx 6.8), \mathrm{CL}+$ $\mathrm{BL}(\approx 6.7), \mathrm{CL}+\mathrm{BS}(\approx 6)$, and $\mathrm{CL}+\mathrm{RS}$ $(\approx 5.5)$ (Table 4). On 11 Sept., AzP and AzP + $\mathrm{BL}$ experienced the highest turfgrass visual quality $(>\approx 7.3)$; followed by CL $(\approx 6)$, AzP + $\mathrm{EO}(\approx 5.8), \mathrm{AzP}+\mathrm{BS}(\approx 5.7), \mathrm{AzP}+\mathrm{RS}$ $(\approx 5.3), \mathrm{CL}+\mathrm{BS}(\approx 4.5), \mathrm{CL}+\mathrm{BS}$ and $\mathrm{CL}+$ $\mathrm{EO}(\approx 3.5)$ (Table 4).

Disease severity. In year 1, no differences occurred on 5 Aug. or 20 Aug. $(>\approx 97 \%)$ (Table 5). On 3 Sept., AzP and CL experienced the lowest disease severity $(\approx 36 \%)$, compared with the reduced synthetic programs $(>\approx 68 \%)$ (Table 5). On 17 Sept., AzP, $\mathrm{CL}$ and $\mathrm{AzP}+\mathrm{BL}($ all $<\approx 11 \%$ ) experienced the lowest disease severity; followed by $\mathrm{CL}+$
$\mathrm{BL}, \mathrm{AzP}+\mathrm{BS}, \mathrm{AzP}+\mathrm{RS}, \mathrm{AzP}+\mathrm{EO}, \mathrm{CL}+$ $\mathrm{BS}, \mathrm{CL}+\mathrm{RS}($ all $<\approx 36 \%$ ), and $\mathrm{CL}+\mathrm{EO}$ $(\approx 75 \%)$ (Table 5). On 1 Oct., AzP + BL, CL, $\mathrm{AzP}, \mathrm{AzP}+\mathrm{BS}, \mathrm{CL}+\mathrm{BL}, \mathrm{CL}+\mathrm{BS}, \mathrm{AzP}+$ $\mathrm{RS}$, and $\mathrm{AzP}+\mathrm{EO}$ experienced the lowest (all $<\approx 15.2 \%$ ) disease severity; followed by $\mathrm{CL}+\mathrm{RS}(\approx 26 \%)$ and $\mathrm{CL}+\mathrm{EO}(\approx 66 \%)$ (Table 5). On 15 Oct., AzP and CL, AzP + $\mathrm{BL}, \mathrm{AzP}+\mathrm{BS}, \mathrm{AzP}+\mathrm{RS}, \mathrm{AzP}+\mathrm{EO}, \mathrm{CL}+$ $\mathrm{BL}$ and $\mathrm{CL}+\mathrm{BS}$ experienced the lowest (all $<\approx 5 \%$ ) disease severity; followed by $\mathrm{CL}+$ $\mathrm{RS}(\approx 12 \%)$ and $\mathrm{CL}+\mathrm{EO}(\approx 43 \%)$ (Table 5$)$.

In year 2 , no differences occurred in disease severity on 1 July or 14 July, with all treatments $>\approx 95 \%$ (Table 6). On 4 Aug., $\mathrm{AzP}(\approx 6 \%)$ and $\mathrm{CL}(\approx 18 \%)$ experienced the lowest disease severity, compared with the reduced synthetic programs $(>\approx 79 \%)$ (Table 6). On 18 Aug., AzP + BL, CL, and AzP experienced lowest (all $<\approx 6 \%$ ) disease severity; followed by AzP + BS ( $\approx 14 \%)$,
$\mathrm{AzP}+\mathrm{EO}(\approx 18 \%), \mathrm{AzP}+\mathrm{RS}(\approx 20 \%), \mathrm{CL}+$ $\mathrm{BL}(\approx 21 \%), \mathrm{CL}+\mathrm{BS}(\approx 32 \%), \mathrm{CL}+\mathrm{RS}$ $(\approx 45 \%)$ and $\mathrm{CL}+\mathrm{EO}(\approx 50 \%)$ (Table 6$). \mathrm{On}$ 31 Aug., AzP, CL, and AzP + BL experienced lowest $(<\approx 3 \%)$ disease severity; followed by $\mathrm{AzP}+\mathrm{BS}(\approx 10 \%), \mathrm{AzP}+\mathrm{EO}$ $(\approx 15 \%), \mathrm{AzP}+\mathrm{RS}(\approx 18 \%), \mathrm{CL}+\mathrm{BL}$ $(\approx 19 \%), \mathrm{CL}+\mathrm{BS}(\approx 24 \%), \mathrm{CL}+\mathrm{RS}$ $(\approx 40 \%)$ and $\mathrm{CL}+\mathrm{EO}(\approx 43 \%)$ (Table 6$)$. On 11 Sept., AzP and AzP + BL experienced lowest $(<10 \%)$ disease severity; followed by $\mathrm{CL}(\approx 18 \%), \mathrm{AzP}+\mathrm{BS}(\approx 35 \%), \mathrm{AzP}+\mathrm{RS}$ $(\approx 45 \%), \mathrm{AzP}+\mathrm{EO}(\approx 54 \%), \mathrm{CL}+\mathrm{BL}$ $(\approx 59 \%), \mathrm{CL}+\mathrm{RS}(\approx 86 \%), \mathrm{CL}+\mathrm{BS}$ $(\approx 91 \%)$ and CL + EO $(\approx 92 \%)$ (Table 6).

Area under disease progress curve. In year $1, \mathrm{AzP}(\approx 241), \mathrm{CL}(\approx 245)$ and $\mathrm{AzP}+$ BL (289) experienced the lowest disease pressure; followed by $\mathrm{CL}+\mathrm{BL}(\approx 308)$, $\mathrm{AzP}+\mathrm{BS}(\approx 310), \mathrm{AzP}+\mathrm{RS}(\approx 328), \mathrm{AzP}$ $+\mathrm{EO}(\approx 329), \mathrm{CL}+\mathrm{BS}(\approx 339), \mathrm{CL}+\mathrm{RS}$ 


\begin{tabular}{ll}
\hline & \multicolumn{1}{c}{ AUDPC $^{\mathrm{z}}$} \\
\cline { 2 - 2 } Treatments & 2008 \\
\hline Azoxystrobin + propiconazole & $240.6 \mathrm{gh}^{\mathrm{y}}$ \\
Azoxystrobin + propiconazole + Bacillus licheniformis & $289.0 \mathrm{efg}$ \\
Azoxystrobin + propiconazole + Reynoutria sachalinensis & $327.6 \mathrm{cde}$ \\
Azoxystrobin + propiconazole + Bacillus subtilis & $309.6 \mathrm{de}$ \\
Azoxystrobin + propiconazole + clove, thyme, and rosemary oil & $329.0 \mathrm{cde}$ \\
Chlorothalonil & $287.3 \mathrm{~h}$ efg \\
Chlorothalonil + Bacillus licheniformis & $378.3 \mathrm{bc}$ \\
Chlorothalonil + Reynoutria sachalinensis & $353.0 \mathrm{bcd}$ \\
Chlorothalonil + Bacillus subtilis & $348.6 \mathrm{bcd}$ \\
Chlorothalonil + clove, thyme, and rosemary oil & $35.0 \mathrm{def}$ \\
\hline
\end{tabular}

${ }^{\mathrm{z}}$ AUDPC was calculated using $\sum\left[\left(\mathrm{Y}_{1}+\mathrm{Y}_{2}\right) / 2\right]\left(\mathrm{T}_{2}-\mathrm{T}_{1}\right)$. Lower values $=$ better control.

${ }^{\mathrm{y}}$ Means with the same letter are not significantly different based on Fisher's least significant difference test $(\alpha=0.05)$.

$(\approx 353)$ and $\mathrm{CL}+\mathrm{EO}(\approx 474)$ (Table 7). In year $2, \mathrm{AzP}, \mathrm{CL}$ and $\mathrm{AzP}+\mathrm{BL}$ experienced lowest $(\approx 210$ to 287 ) disease pressure; followed by $\mathrm{AzP}+\mathrm{EO}(\approx 349)$, AzP $+\mathrm{BS}$ $(\approx 353), \mathrm{AzP}+\mathrm{RS}(\approx 378), \mathrm{CL}+\mathrm{BL}$ $(\approx 385), \mathrm{CL}+\mathrm{BS}(\approx 447), \mathrm{CL}+\mathrm{RS}(\approx 450)$, and CL + EO $(\approx 497)$ (Table 7).

\section{Discussion}

Compared with a previous trial, programs containing one-quarter rates of synthetic fungicides in combination with the BCAs, provided increased efficacy (Lo et al., 1997). Previously, synthetics + BCAs were used on a 30-day interval (Lo et al., 1997) compared with the 14-day interval in the present study, possibly indicating proper timing of synthetic products during increased disease pressure. Previous research indicates applications of BCAs provided both rhizosphere and foliar inoculation (Lo et al., 1997). Weekly applications of BCAs were able to provide control similar to that by synthetic fungicides. Monthly spray applications of BCAs were able to reduce DS; however, these did not achieve control similar to weekly applications. Research conducted at Purdue University during the spring indicated similar trends. Reduced rates of $\mathrm{CL}+\mathrm{BCAs}$ achieved similar control as full labeled rates of CL (Latin, 2008). Treatments at the Purdue University study were applied on either a 7or 14-day interval, whereas treatments during the present study were all applied on a 14-day interval. Bowers and Locke (2000) reported reductions in Fusarium oxysporum populations when clove oil was applied. Improved turfgrass visual quality was also noted with $\mathrm{AzP}$ and BL during both years of this study. Marvin et al. (2020) reported reduced label rates of $\mathrm{CL}$ and $\mathrm{AzP}$ in combination with BCAs improved DS control during in vitro and preventive field trials. Further greenhouse evaluations should be conducted to determine the possible physiological effects these fungicides are having on the plant, leading to enhanced color. Due to earlier disease pressure, the second study year was initiated earlier than the first year. Disease pressure increased at the end of year 2 . Treatments were stopped earlier than the first year to evaluate any "rebound effect" in disease pressure. Treatments were stopped on 17 Sept. in year 1 and 18 Aug. in year 2. From 31 Aug. to 11 Sept. in year 2, an increase in disease severity was noted. In general, continued applications may be required until environmental conditions are no longer conducive to DS outbreaks.

Previous research evaluating environmental impact quotient of BCAs revealed less impact to the environment than conventional synthetic programs (Grant and Rossi, 2006).

In conclusion, all reduced programs, except CL + EOs, provided acceptable disease severity $(\leq 15 \%)$ at the end of year 1 and acceptable $(\geq 7)$ turfgrass visual quality. Results from year 1 are similar to TomasoPeterson (2006), where control was achieved using BCAs when disease pressure was not severe. In year 2, AzP, CL, AzP + BL, AzP + $\mathrm{EO}$, and $\mathrm{AzP}+\mathrm{BS}$ all provided $\leq 15 \%$ disease severity and $\geq 7$ visual turfgrass quality $14 \mathrm{~d}$ after the last application. At $28 \mathrm{~d}$ after the last application in year 2, an increase in disease severity occurred. The only treatments to maintain adequate DS control and visual turfgrass quality ratings were $\mathrm{AzP}$ and $\mathrm{AzP}+\mathrm{BL}$. The ability of $\mathrm{AzP}+$ $\mathrm{BL}$ to maintain acceptable turf quality is a promising step in developing programs with reduced amounts of synthetic products, while providing similar control. Future research should focus on understanding the competitive relationship between BCAs and turfgrass pathogens. Understanding the growth and development dynamics between the BCAs and turfgrass pathogens will lead to improved control efficacy.

\section{Literature Cited}

Bishop, P., J. Sorochan, B.H. Ownley, T.J. Samples, A.S. Windham, M.T. Windham, and R.N. Trigiano. 2008. Resistance of Sclerotinia homoeocarpa to iprodione, propiconazole, and thiophanate-methyl in Tennessee and Northern Mississippi. Crop Sci. 48:16151620.
Bowers, J.H. and J.C. Locke. 2000. Effect of botanical extracts on the population density of Fusarium oxysporum in soil and control of Fusarium wilt in the greenhouse. Plant Dis. 84(3): 300-305.

Grant, J.A. and F.S. Rossi. 2006. Long-term evaluation and improvement of golf turf management systems with reduced chemical pesticide inputs: Preliminary Report. Ithaca, NY. Cornell University. <https://hdl.handle.net/1813/ 43166>.

Harman, G.E. 2006. Overview of mechanisms and uses of Trichoderma spp. Phytopathology 96:190-194.

Kim, D.S, R.J. Cook, and D.M. Weller. 1997. Bacillus sp. L324-92 for biological control of three root diseases of wheat grown with reduced tillage. Phytopathology 87:551-558.

Latin, R. 2008. Interaction of biofungicides and chlorothalonil for control of dollar spot on creeping bentgrass. Report. Purdue University, West Lafayette, IN.

Lo, C.T., E.B. Nelson, and G.E. Harman. 1997. Improved biocontrol efficacy of Trichoderma harzianum 1295-22 for foliar phases of turf diseases by use of spray applications. Plant Dis. 81:1132-1138.

Marvin, J.W., R.A. Kerr, L.B. McCarty, W.C. Bridges, S.B. Martin, and C.E. Wells. 2020. In vitro and preventative field evaluations of potential biological control agents and synthetic fungicides for control of Clarireedia jacksonii sp. nov. J. Plant Sci. Phytopathol. 4:001-008.

McCarty, L.B. 2018. Golf turf management. CRC Press, Boca Raton, FL.

Morris, K.N. and R.C. Shearman. 1999. NTEP turfgrass evaluation guidelines. National Turfgrass Evaluation Program, Beltsville, MD.

Salgado-Salazar, C., L.A. Beirn, A. Ismaiel, M.J. Boehm, I. Carbone, A.I. Putman, L.P. Tredway, B.B. Clarke, and J.A. Crouch. 2018. Clarireedia: A new fungal genus comprising four pathogenic species responsible for dollar spot disease of turfgrass. Fungal Biol. 122(8):761773.

Smiley, R.W., P.H. Dernoeden, and B.B. Clarke. 2005. Compendium of turfgrass diseases. 3rd ed. APS Press, Saint Paul, MN.

Tomaso-Peterson, M. 2006. A demonstration trial of biofungicides with efficacy for controlling dollar spot in turfgrass. Mississippi Agricultural \& Forestry Experiment Station. (23)17. 